Research Article

\title{
Self-Sustained Oscillation of a Photothermal-Responsive Pendulum under Steady Illumination
}

\author{
Dali Ge $\mathbb{D}^{1},{ }^{1,2}$ Peibao Xu $\mathbb{D}^{1},{ }^{1}$ and Kai Li $\mathbb{D}^{1}$ \\ ${ }^{1}$ Department of Civil Engineering, Anhui Jianzhu University, Hefei 230601, Anhui, China \\ ${ }^{2}$ Institute of Advanced Technology, University of Science and Technology of China, Hefei 230001, Anhui, China \\ Correspondence should be addressed to Kai Li; kli@ahjzu.edu.cn
}

Received 8 January 2021; Revised 28 January 2021; Accepted 3 February 2021; Published 16 February 2021

Academic Editor: Sundarapandian Vaidyanathan

Copyright $(2021$ Dali Ge et al. This is an open access article distributed under the Creative Commons Attribution License, which permits unrestricted use, distribution, and reproduction in any medium, provided the original work is properly cited.

Self-sustained oscillation has the advantages of harvesting energy from the environment and self-control, and thus, the development of new self-oscillating systems can greatly expand its applications in active machines. In this paper, based on conventional photothermal shrinkable material or photothermal expansive material, a simple pendulum is proposed. The lightpowered self-sustained oscillation of the simple pendulum is theoretically studied by establishing a dynamic model of the photothermal-responsive pendulum. The results show that there are two motion modes of the simple pendulum, which are the static mode and the oscillation mode. Based on the photothermal-responsive model, this paper elucidates the mechanism of the self-excited oscillation. The condition for triggering self-excited oscillation is further studied. In addition, the influence of the system parameters on the amplitude and frequency is also obtained. This study may have potential applications in energy harvesting, signal monitoring, and soft machines.

\section{Introduction}

Photothermal-responsive materials exhibit photothermal effects under light illumination, which results in some macroscopic changes, such as shape change and color change. Due to the advantages of light stimulus, such as environmentally friendly, remote controllability, and instantaneity, photothermal-responsive materials have attracted many researchers' attention. Suzuki and Tanaka [1] introduced the photosensitive component chlorophyll in the gel and designed a light-sensitive gel. The temperature of the illuminated gel increases and the gel shrinks. Hu et al. [2] found that PNIPAM gel will reduce the transmittance of visible laser light when infrared radiation makes the gel locally heated up. Kubo et al. [3] filled silicon dioxide inverse opal with liquid crystal molecules and made a device that changes the color of reflected light caused by the light response.

Photothermal-responsive materials with reasonable design can produce photo-induced deformation and motion for a variety of flexible intelligent actuators, which have wide application prospects in the fields of artificial muscles [4], retractable optical devices [5], light-driven microbionic propellers [6-8], light-powered microfluidic pump [9], lightfueled microgenerator [10], and so on. Ikeda et al. [11] used alternating ultraviolet and visible light to achieve cis-trans isomer photomechanical actuation of liquid crystal elastomers containing azobenzene. Carbon nanotubes, graphene, and graphene oxide have good photothermal effects, which can convert near-infrared light and visible light into heat [12-15]. They can be used as nanoheaters to generate local heat and cause conformational modification or phase transition of elastomer matrix [15-18] to realize lightpowered motions.

Self-excited oscillations have been observed in many fields of science and engineering [19-23] and can be utilized to produce periodic motions which are often required in numerous engineering applications [24-28]. Different from the forced vibration with damping dissipation, which often applies periodic stimulus to cause periodic vibration, the self-excited oscillation utilizes the nonlinear feedback within the system to result in and maintain steady-state periodic 
motions, such as the flapping of leaves under the breeze, the peristalsis of the digestive tract, the periodic beating of the heart, and the beautiful music produced by the violin bow. Under the steady environment, the self-excited oscillation system takes its own motion state as the regulator to balance the input energy and the dissipated energy, so as to realize the continuous periodic motion. The characteristics of the self-excited oscillation system, such as frequency and amplitude, are often determined by the physical and geometrical parameters of the system and have nothing to do with the initial conditions. The particularity of the self-excited oscillation arouses the interest of researchers and has been applied in robots and other fields. Xu et al. [24] have carried out dynamic simulation for a woodpecker robot running under friction and collision. Ono et al. [25] studied the mechanism of achieving steady walking motion of a selfoscillating bipedal knee robot. Morazzani et al. [26] proposed a new type of self-excited three-legged walking robot. According to the principle of bionics, Li et al. [27] proposed a pipeline crawling microrobot based on the self-excited oscillation. Yamano et al. [28] constructed a multilink adaptive control swimming robot model, which can generate different self-excited oscillations according to the change of environment, so as to change the motion mode.

Recently, the self-excited oscillation of optomechanical oscillators has been extensively explored and applied in macrodevices [29], microdevices [30], and nanodevices [31]. There exist various self-excited periodic motions in systems dominated by photothermal effects $[32,33]$. With the in-depth study of photothermal-responsive materials, more progress has been made in the study of self-excited oscillation based on photothermalresponsive materials. Hollander et al. [34] studied a nonlinear thermoviscoelastic cantilever coated with a light-absorbing film. The self-excited oscillation of the cantilever is triggered by the photothermal effect. Dirk J. Gelebart's research group [35] added light-absorbing dyes to liquid crystal network polymers and prepared a continuous oscillating composite film by the photothermal effect. Houri et al. [36] irradiate the graphene barrel with a red continuous wave laser beam, and it oscillates autonomously by photothermal feedback. Buks and Martin [37] studied a fully on-fiber optomechanical cavity which was formed by patterning a suspended metallic mirror near the tip of an optical fiber and by introducing a static reflector inside the fiber and characterized its performance as a sensor. Optically induced self-excited oscillation is observed above a threshold value of the injected laser power.

Although some self-excited oscillations of photothermalresponsive materials and their applications have been studied, the modes of self-excited oscillation are not enough, which limits the potential applications of self-excited oscillation. In this paper, a simple pendulum is constructed by using conventional photothermal expansive material (PEM) or photothermal shrinkable material (PSM) as a cycloid, and the governing equation of variable pendulum length is established based on the photothermal effect. Through numerical calculation, it is verified that the photothermal-responsive pendulum can realize self-excited oscillation under steady light illumination. The mechanism of self-excited oscillation is elucidated, the conditions for realizing self-excited oscillation are discussed, and the dependence of amplitude and frequency on system parameters is extensively studied.

\section{Theoretical Model and Formulation}

2.1. Governing Equations for Dynamics of the Simple Pendulum. The dynamic model and governing equations of a photothermal-responsive pendulum under steady illumination are established in this section. As shown in Figure 1, a simple pendulum made of photothermal-responsive materials is established and illuminated steadily. The end of the cycloid made of photothermal-responsive material with negligible mass is tied to a fixed point $O$. A pendulum ball with mass $m$ is suspended by the cycloid. By establishing a polar coordinate system, the position of a simple pendulum can be expressed by pendulum length $r$ and swing angle $\theta$. During the motion of the pendulum, the length $r$ and swing angle $\theta$ of the pendulum both vary with the time. In the polar coordinate system, two mutually perpendicular unit vectors $\mathbf{e}_{r}$ and $\mathbf{e}_{\theta}$ are along the directions of increasing pendulum length and pendulum angle, respectively, and they also change during the swing of the pendulum.

To obtain the self-excited motion, a PSM pendulum should be illuminated in the middle zone as shown in Figure 1(a), while a PSE pendulum should be illuminated on the two sides as shown in Figure 1(b), where the angle $\varphi$ denotes the illuminated zone or dark zone in the middle. For the two pendulum systems in Figure 1, the cycloid gradually shrinks when it is in the middle zone, while gradually expands when it swings out of the middle zone. Eventually, the pendulum could swing continuously as discussed as follows.

In the polar coordinate system, the velocity and acceleration of the pendulum along the directions $\mathbf{e}_{\theta}$ and $\mathbf{e}_{r}$ are as follows:

$$
\begin{aligned}
& v_{\theta}=r \dot{\theta}, \\
& a_{\theta}=r \theta+2 \dot{r} \dot{\theta}, \\
& v_{r}=\dot{r}, \\
& a_{r}=r-r \dot{\theta}^{2},
\end{aligned}
$$

where $\dot{r}$ and $\ddot{r}$ are the first derivative $\mathrm{d} r / \mathrm{d} t$ and the second derivative $\mathrm{d}^{2} r / \mathrm{d} t^{2}$, respectively, and $\dot{\theta}$ and $\ddot{\theta}$ are the first derivative $\mathrm{d} \theta / \mathrm{d} t$ and the second derivative $\mathrm{d}^{2} \theta / \mathrm{d} t^{2}$, respectively.

As shown in Figure 1, $\mathrm{mg}$ is the gravity of the pendulum, $F$ is the tension of the cycloid, and $F_{\theta}$ and $F_{r}$ are the components of the air damping force in $\mathbf{e}_{\theta}$ and $\mathbf{e}_{r}$ directions, respectively. Since the fiber is generally much thinner than the pendulum ball, the air damping force of the fiber is ignored for simplicity. It is assumed that the air damping force of the pendulum is proportional to the velocity of the ball, and the direction is opposite to the velocity of the ball. The governing equation for the dynamic of the pendulum can be given as 


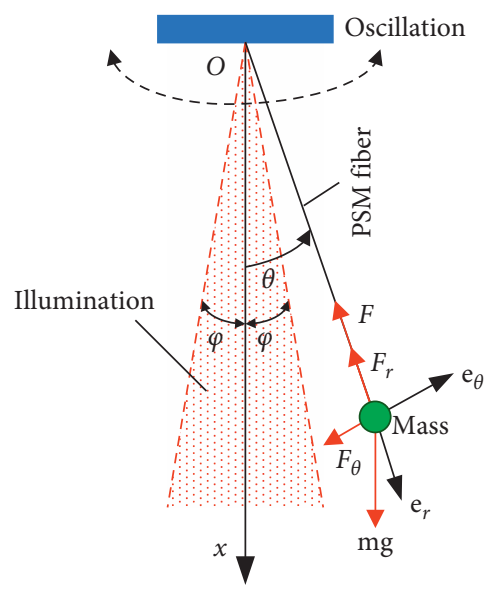

(a)

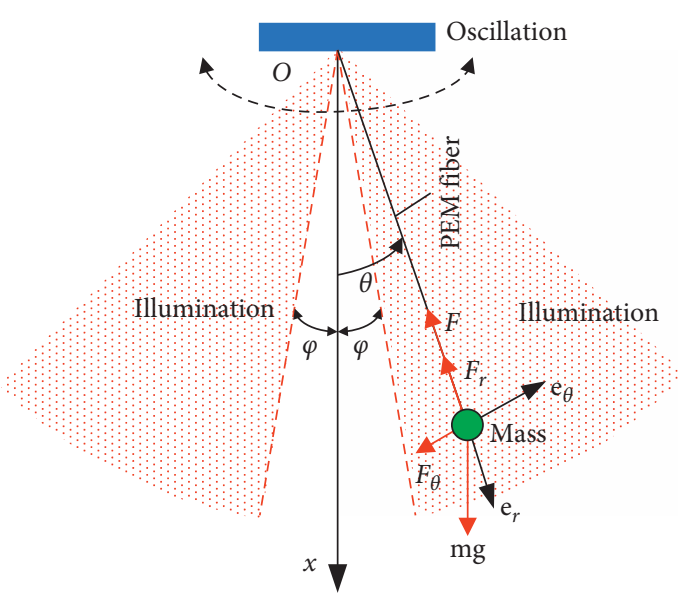

(b)

FIGURE 1: Schematics of the light-driven swing of a photothermal-responsive pendulum based on (a) PSM cycloid and (b) PEM cycloid.

$$
\begin{aligned}
& m(r \ddot{\theta}+2 \dot{r} \dot{\theta})=-\mathrm{mg} \sin \theta-\beta \dot{\theta} r, \\
& m\left(\ddot{r}-r \dot{\theta}^{2}\right)=\mathrm{mg} \cos \theta-\beta \dot{r}-F,
\end{aligned}
$$

where $\beta$ is the linear air damping coefficient. To study the light-powered motion of a simple pendulum with the photothermal response and its mechanism, equation (2) is rewritten as

$$
\begin{aligned}
& \ddot{\theta}=-\left(\frac{2 \dot{r}}{r}+\frac{\beta}{m}\right) \dot{\theta}-\frac{g}{r} \sin \theta, \\
& F=m g \cos \theta-\beta \dot{r}-m\left(\ddot{r}-r \dot{\theta}^{2}\right) .
\end{aligned}
$$

2.2. Model of the Photothermal-Responsive Pendulum. This section mainly describes the dynamics of temperature and length of the photothermal-responsive pendulum under stable light illumination and in dark. Since the simple pendulums made with PSM and PEM in Figure 1 have the same photothermal effect, in the following, the simple pendulum of PSM in Figure 1(a) is chosen as the representative of the photothermal-responsive pendulum.

The cycloid of the pendulum has a very small radius, i.e., the well-known Biot number is very small. Therefore, heat exchange in the pendulum is assumed to be very fast and the temperature in the pendulum is homogeneous. Due to the photothermal effect, the pendulum can convert light illumination into heat. The heat converted from light per second is denoted by $q$. The pendulum can also exchange heat with the environment, and the heat flux is assumed to be linear to the temperature difference $T$ between the pendulum and the environment. Under light illumination, the temperature difference $T$ is determined by

$$
\dot{T}=\frac{q-k T}{\rho_{c}},
$$

where $\rho_{c}$ is the specific heat capacity and $k$ is the heat transfer coefficient. By solving equation (4), in the illumination zone, the temperature difference $T$ is

$$
T=T_{0}\left(1-e^{-t / \tau}\right)
$$

while in the dark zone, i.e., $q=0$, the temperature difference $T$ is

$$
T=T_{0} e^{-t / \tau}
$$

where $T_{0}=q / k$ represents the limit temperature difference of photothermal-responsive fiber under longtime illumination, $\tau=\rho_{c} / k$ reflects the characteristic time for heat exchange between photothermal-responsive fiber and environment, and the larger $\tau$ indicates the longer time required for attaining the limit temperature difference $T_{0}$ of the photothermal-responsive fiber.

Deformation of the photothermal-responsive pendulum can be driven by the temperature change, and the thermal strain of the fiber is assumed to be linear to the temperature change. The elastic modulus is assumed to be very large, and the elastic strain is ignored. Therefore, the length of the PSM fiber can be calculated as, in the illumination zone:

$$
r=r_{0}\left[1-\alpha T_{0}\left(1-e^{-t / \tau}\right)\right]
$$

in the dark zone:

$$
r=r_{0}\left(1-\alpha T_{0} e^{-t / \tau}\right)
$$

where $r_{0}$ is the origin length of the PSM fiber without thermal strain and $\alpha$ is the linear thermal contraction coefficient of the PSM fiber.

2.3. Nondimensionalization. To nondimensionalize the governing equations above, we introduce the following dimensionless parameters: $\bar{t}=t / \tau, \bar{T}=T / T_{0}, \quad \bar{r}=r / r_{0}$, $\varepsilon_{0}=\alpha T_{0}, \bar{\beta}=\beta \tau / m, \bar{g}=g \tau^{2} / r_{0}$, and $\bar{F}=F \tau^{2} / m r_{0}$. Here, $\varepsilon_{0}$ is the contraction strain of the photothermal-responsive cycloid at the limit temperature difference $T_{0}$, and $\bar{\beta}$ reflects the 
air damping. $\bar{g}$ can be rewritten as $\bar{g}=\left(\tau / \tau_{0}\right)^{2}$ by the natural period $\tau_{0}=\sqrt{r_{0} / g}$ of the single pendulum, so it represents the speed of heat transfer relative to natural oscillation. The larger $\bar{g}$ is, the faster the heat transfers. The dimensionless parameter $\bar{F}$ characterizes the tension of the photothermalresponsive cycloid. Then, equations (5)-(8) can also be rewritten as, in the illumination zone, i.e., $-\varphi \leq \theta \leq \varphi$.

$$
\begin{aligned}
& \bar{T}=1-e^{-\bar{t}}, \\
& \bar{r}=1-\varepsilon_{0}\left(1-e^{-\bar{t}}\right) .
\end{aligned}
$$

in the dark zone, i.e., $\theta<-\varphi$ or $\theta>\varphi$ :

$$
\begin{aligned}
\bar{T} & =e^{-\bar{t}}, \\
\bar{r} & =1-\varepsilon_{0} e^{-\bar{t}} .
\end{aligned}
$$

The following differential equation is introduced:

$$
\begin{aligned}
& \dot{r}=\frac{\mathrm{d} r}{\mathrm{~d} t}=\frac{1}{\tau} \frac{\mathrm{d} r}{\mathrm{~d} \bar{t}}=\frac{\dot{\bar{r}}}{\tau}, \\
& r=\frac{\mathrm{d}^{2} r}{\mathrm{~d} t^{2}}=\frac{\ddot{\bar{r}}}{\tau^{2}} .
\end{aligned}
$$

By inserting equations (9)-(11), equation (3) can be derived as, in the illumination zone, i.e., $-\varphi \leq \theta \leq \varphi$ :

$$
\begin{aligned}
\frac{\mathrm{d}^{2} \theta}{\mathrm{d} \bar{t}^{2}} & =\left[\frac{2 \varepsilon_{0} e^{\bar{t}}}{1-\varepsilon_{0}\left(1-e^{\bar{t}}\right)}-\bar{\beta}\right] \frac{\mathrm{d} \theta}{\mathrm{d} \bar{t}}-\frac{\bar{g}}{1-\varepsilon_{0}\left(1-e^{\bar{t}}\right)} \sin \theta, \\
\bar{F} & =\bar{g} \cos \theta+\bar{\beta} \varepsilon_{0} e^{-\bar{t}}-\varepsilon_{0} e^{-\bar{t}}+\left[1-\varepsilon_{0}\left(1-e^{-\bar{t}}\right)\right]\left(\frac{\mathrm{d} \theta}{\mathrm{d} \bar{t}}\right)^{2},
\end{aligned}
$$

in the dark zone, i.e., $\theta<-\varphi$ or $\theta>\varphi$ :

$$
\begin{aligned}
\frac{\mathrm{d}^{2} \theta}{\mathrm{d} \bar{t}^{2}} & =\left[\frac{-2 \varepsilon_{0} e^{\bar{t}}}{1-\varepsilon_{0} e^{\bar{t}}}-\bar{\beta}\right] \frac{\mathrm{d} \theta}{\mathrm{d} \bar{t}}-\frac{\bar{g}}{1-\varepsilon_{0} e^{\bar{t}}} \sin \theta, \\
\bar{F} & =\bar{g} \cos \theta-\bar{\beta} \varepsilon_{0} e^{-\bar{t}}+\varepsilon_{0} e^{-\bar{t}}+\left(1-\varepsilon_{0} e^{-\bar{t}}\right)\left(\frac{\mathrm{d} \theta}{\mathrm{d} \bar{t}}\right)^{2} .
\end{aligned}
$$

2.4. Solution Method. The second-order differential equations (12) and (13) provide a complete description of the dynamics of the photothermal-responsive pendulum. The swing angle $\theta$ can be obtained by the first equation in equation (13), and then, the pendulum force $\bar{F}$ can be further obtained by the second equation in equation (13). It is very challenging to obtain an analytical solution to equation (13). In this paper, we will numerically solve the swing of the pendulum based on the well-known fourth-order Runge-Kutta method. By letting $\psi=\dot{\theta}$, the second-order ordinary differential equation (13) is transformed into a first-order differential equation system as follows:

$$
\frac{\mathrm{d} \theta}{\mathrm{d} \bar{t}}=\psi,
$$

in the illumination zone, i.e., $-\varphi \leq \theta \leq \varphi$ :

$$
\frac{\mathrm{d} \psi}{\mathrm{d} \bar{t}}=\left[\frac{2 \varepsilon_{0} e^{\bar{t}}}{1-\varepsilon_{0}\left(1-e^{\bar{t}}\right)}-\bar{\beta}\right] \psi-\frac{\bar{g}}{1-\varepsilon_{0}\left(1-e^{\bar{t}}\right)} \sin \theta,
$$

in the dark zone, i.e., $\theta<-\varphi$ or $\theta>\varphi$ :

$$
\frac{\mathrm{d} \psi}{\mathrm{d} \bar{t}}=\left[\frac{-2 \varepsilon_{0} e^{\bar{t}}}{1-\varepsilon_{0} e^{\bar{t}}}-\bar{\beta}\right] \psi-\frac{\bar{g}}{1-\varepsilon_{0} e^{\bar{t}}} \sin \theta .
$$

The initial conditions are $\left.\theta\right|_{\bar{t}=0}=\theta_{0}$ and $\left.\psi\right|_{\bar{t}=0}=\dot{\theta}_{0}$. Given the dimensionless parameters $\varphi, \bar{\beta}, \varepsilon_{0}$, and $\bar{g}$, the solution to equations (14)-(16) can be obtained numerically by programming in software Matlab. When the angle $\theta(\bar{t})$ and the angular velocity $\dot{\theta}(\bar{t})$ of the simple pendulum are obtained, $\bar{T}(\bar{t}), \bar{r}(\bar{t})$, and $\bar{F}(\bar{t})$ can be further calculated.

\section{Two Motion Modes and Mechanism of the Self-Excited Oscillation}

3.1. Two Motion Modes. By setting the parameters $\theta_{0}, \dot{\theta}_{0}, \varphi, \bar{\beta}$, $\varepsilon_{0}$, and $\bar{g}$, the time histories and phase diagrams of the lightpowered swing of the pendulum can be obtained. The numerical calculation shows that there exist two motion modes of the simple pendulum: static mode and oscillation mode, as shown in Figure 2 . In the computation, we set $\theta_{0}=0, \dot{\theta}_{0}=0.2$, $\varphi=0.1, \bar{\beta}=0.12$, and $\bar{g}=2$. For $\varepsilon_{0}=0$, the pendulum with initial angular velocity first swings, and then, the amplitude of swing gradually decreases under the air damping, as shown in Figures 2(a) and 2(b). Eventually, the pendulum comes to cease at the middle, which is named as static mode. Although the pendulum is illuminated and the cycloid temperature rises, the pendulum length for $\varepsilon_{0}=0$ will not change and cannot convert light energy into kinetic energy. For $\varepsilon_{0}=0.3$, the pendulum under light illumination can swing continuously and finally develops into self-sustained oscillation, which is named as oscillation mode (Figures 2(c) and 2(d)). Although there is damping in the system, the energy compensation from the light maintains the oscillation. In the following section, we further explore the mechanism of self-excited oscillation and in detail elucidate how the light energy converts into kinetic energy.

3.2. Mechanism of the Self-Excited Oscillation. To explore the mechanism of self-excited oscillation, the evolution laws of the temperature and length of the cycloid are first studied. Based on equations (9) and (10), Figure 3 plots the evolution law of $\bar{T}$ and $\bar{r}$ during swing in the illumination zone and the dark zone. As shown in Figure 3(a), the temperature of cycloid increases under continuous illumination, and the dimensionless temperature difference parameter $\bar{T}$ can gradually increase from 0 to nearly 1 . In the dark zone, $\bar{T}$ can gradually decrease from the maximum value of 1 to 0 . When the pendulum passes through the interface of the illumination and dark zones, the temperature of the cycloid will 


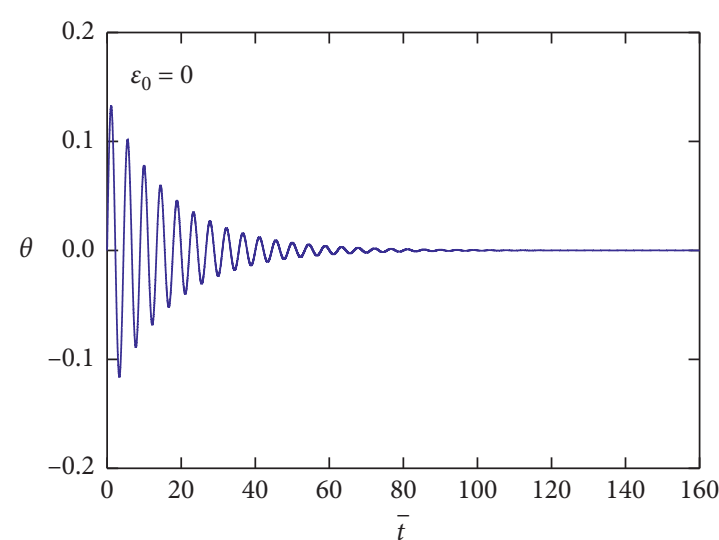

(a)

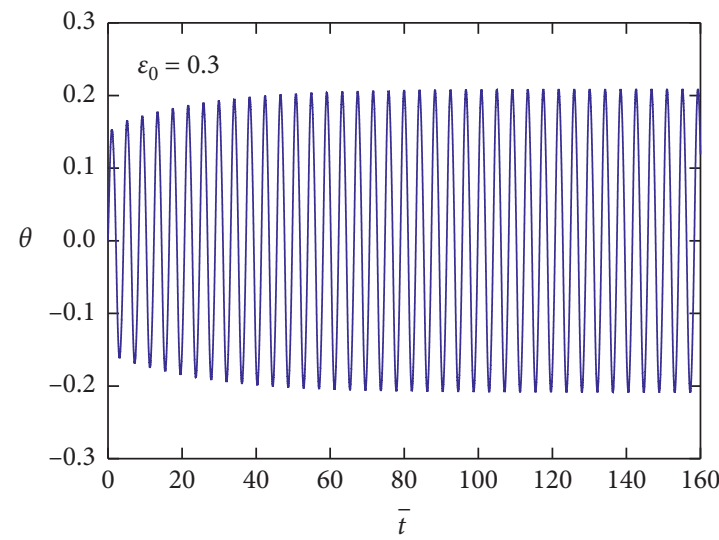

(c)

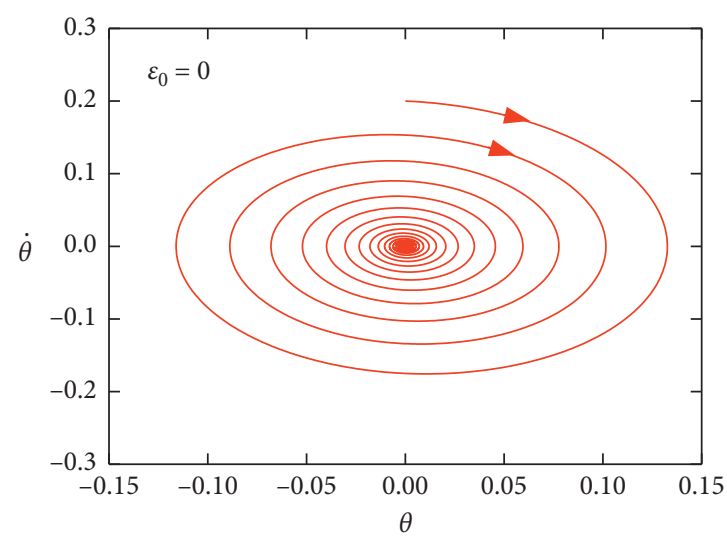

(b)

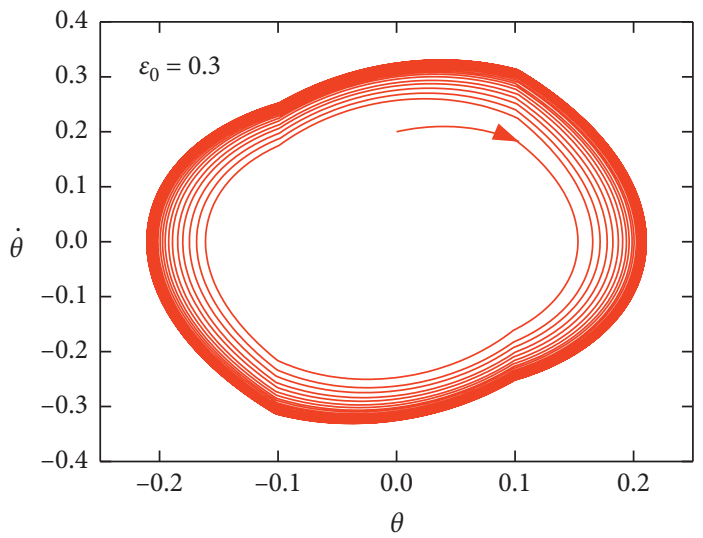

(d)

Figure 2: Time histories and phase diagrams of two motion modes of the pendulum: (a,b) the static mode for $\varepsilon_{0}=0$; (c, d) the oscillation mode for $\varepsilon_{0}=0.3$.

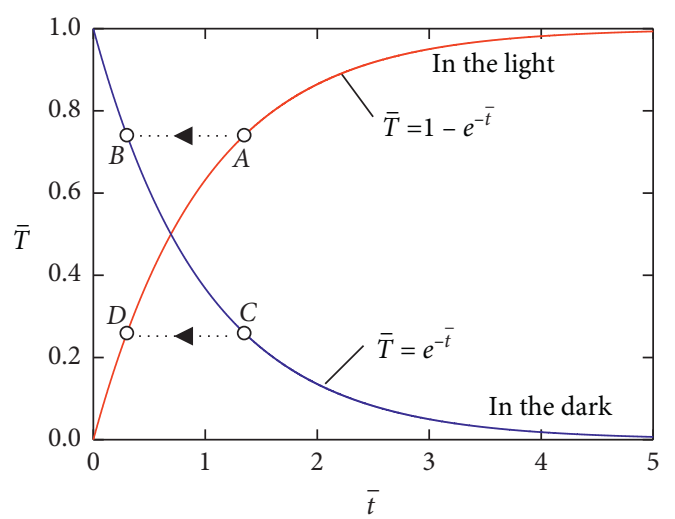

(a)

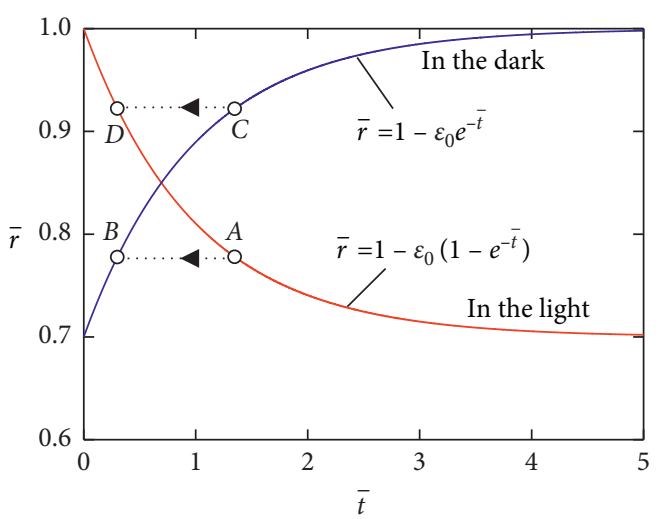

(b)

FIgURe 3: Evolution laws of (a) $\bar{T}$ and (b) $\bar{r}$ of the PSM pendulum.

switch to obeying the other evolution law, i.e., from point $A$ to point $B$ or from point $C$ to point $D$ as shown in Figure 3(a). Similarly, the evolution law of the dimensionless parameter $\bar{r}$ for $\varepsilon_{0}=0.3$ in Figure $3(\mathrm{~b})$ shows that the pendulum length $\bar{r}$ contracts into $1-\varepsilon_{0}$ in the illumination zone, while recovers gradually to 1 in the dark zone. The pendulum length also switches when the pendulum passes through the interface between the illumination and dark zones.

Based on the evolution law in Figure 3, the mechanism of the self-excited oscillation in Figure 2(b) can be elucidated by investigating the time histories of $\bar{T}$ and $\bar{r}$ in detail. Figure 4(a) plots the dependence of the dimensionless temperature difference parameter $\bar{T}$ on the swing angle $\theta$. 


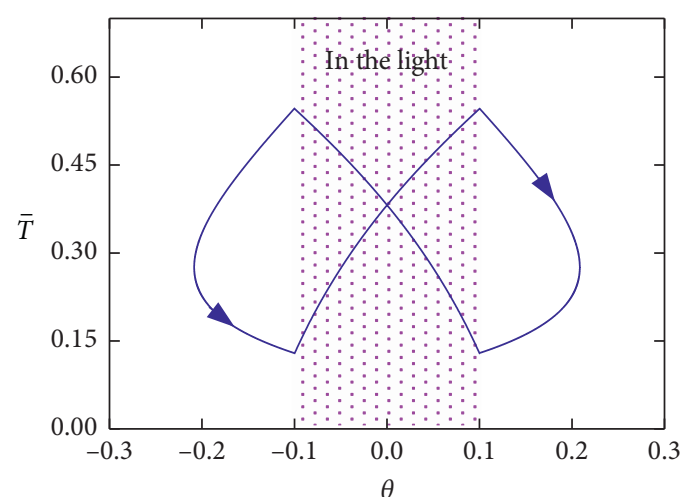

(a)

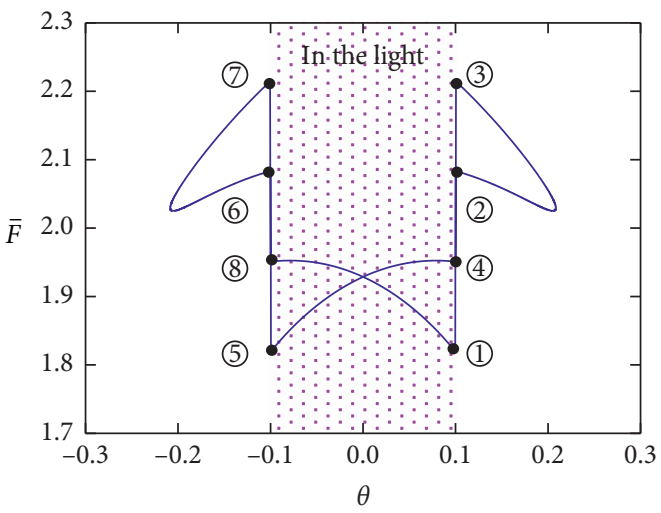

(c)

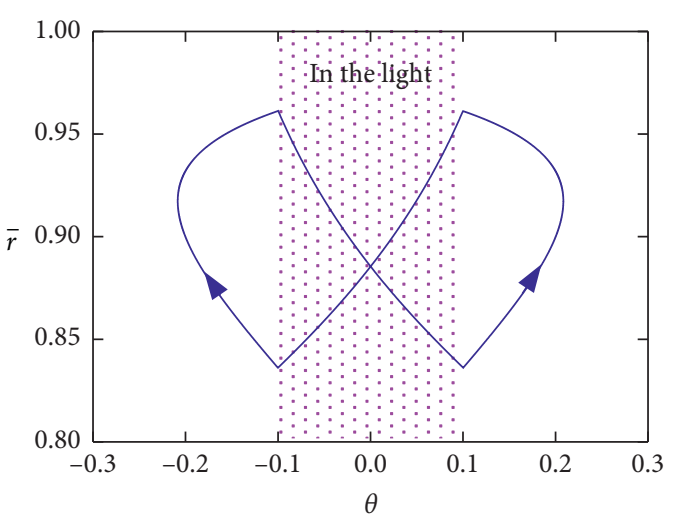

(b)

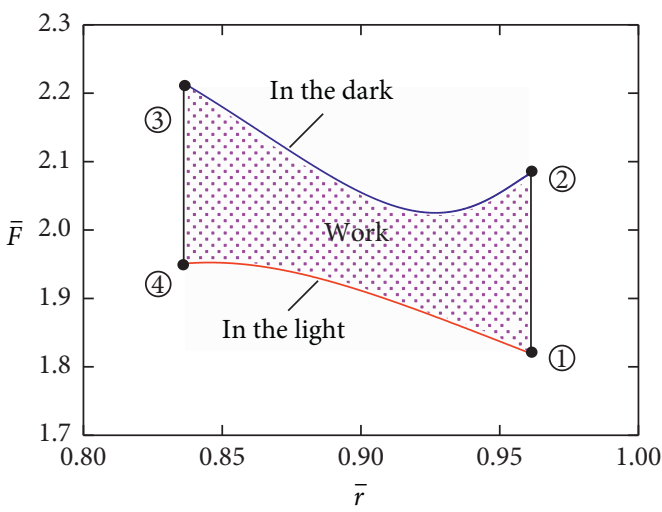

(d)

FIGURE 4: Mechanism of self-excited oscillation of the PSM pendulum, for $\theta_{0}=0, \dot{\theta}_{0}=0.2, \varphi=0.1, \bar{\beta}=0.12, \varepsilon_{0}=0.3$, and $\bar{g}=2$. (a) $\bar{T}$ vs. $\theta$. (b) $\bar{r}$ vs. $\theta$. (c) $\bar{F}$ vs. $\theta$. (d) $\bar{F}$ vs. $\bar{r}$.

For $-0.1 \leq \theta \leq 0.1$, the cycloid is in the illumination zone and $\bar{T}$ increases. When the pendulum swings into the dark zone, $\bar{T}$ decreases. Similarly, Figure 4(b) plots the dependence of $\bar{r}$ on $\theta$. The length $\bar{r}$ decreases in the illumination zone, while it increases in the dark zone. Further, Figure 4(c) plots the dependence of $\bar{F}$ on $\theta$, where $\bar{F}$ is discontinuous at some points of $\theta$. The tension $\bar{F}$ sharply increases at the moment of swinging into the dark region (point (2) from the illumination region (point (1)). This is because the temperature of the pendulum swinging into the dark zone decreases and in turn the cycloid length increases. As the pendulum swings in the dark area, $\bar{F}$ continuously varies from point (2) to (3). Then, the tension $\bar{F}$ sharply decreases from point (3) to point (4) when the pendulum reenters into the illumination zone. Eventually, tension $\bar{F}$ and swing angle $\theta$ form a symmetrical closed loop according to the trajectory cycle of (1) (2) (3) (4) (5) (6) (7) (8) (1) in a swing period.

Based on Figures 4(a)-4(c), Figure 4(d) plots the dependence of $\bar{F}$ with $\bar{r}$, where the shadow area enclosed by curve of $\bar{F}$ with $\bar{r}$ represents the magnitude of the work done by the tension $\bar{F}$ in a half swing period of the simple pendulum. In an oscillation period, the work done by $\bar{F}$ is twice shadow area in Figure 4(d), which is the source of selfexcited oscillation. When the net work is enough for compensating the dissipation by air damping, the pendulum can oscillate continuously.

\section{Influence of Systematic Parameters on the Self-Excited Oscillation}

In equations (15) and (16), there are six dimensionless parameters: $\varphi, \bar{\beta}, \varepsilon_{0}, \bar{g}, \theta_{0}$, and $\dot{\theta}_{0}$. The swing of the PSM pendulum in Figure 1(a) is controlled by the above six parameters. In this section, the influence of the six parameters on the onset, frequency, and amplitude of the selfexcited oscillation is extensively investigated. Considering the parameter $\theta_{0}$ can be transformed into the corresponding value $\dot{\theta}_{0}$ through the energy transformation between gravitational energy and kinetic energy, $\theta_{0}$ is set to be zero in the calculation. The dimensionless oscillation frequency $\bar{f}$ of the simple pendulum is defined as the number of oscillations per unit time $\bar{t}$, and the angular amplitude of the self-excited oscillation of the simple pendulum is denoted by $A$.

4.1. Effect of Initial Angular Velocity $\dot{\theta}_{0}$. Figure 5 shows the influence of the initial angular velocity $\dot{\theta}_{0}$ on the swing of the simple pendulum, for $\theta_{0}=0, \varphi=0.1, \bar{\beta}=0.12, \varepsilon_{0}=0.3$, and $\bar{g}=2$. Figure $5(\mathrm{a})$ plots the phase diagram of the swing angle for $\dot{\theta}_{0}=0.12$. The result shows that the simple pendulum swings with gradually attenuating amplitude and eventually comes to rest, which is static mode. The maximum swing angle $\theta_{\max }$ of simple pendulum is less than $\varphi$ in the initial 


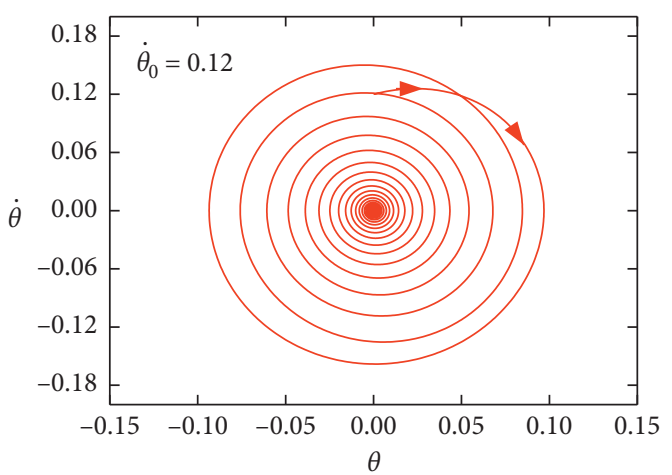

(a)

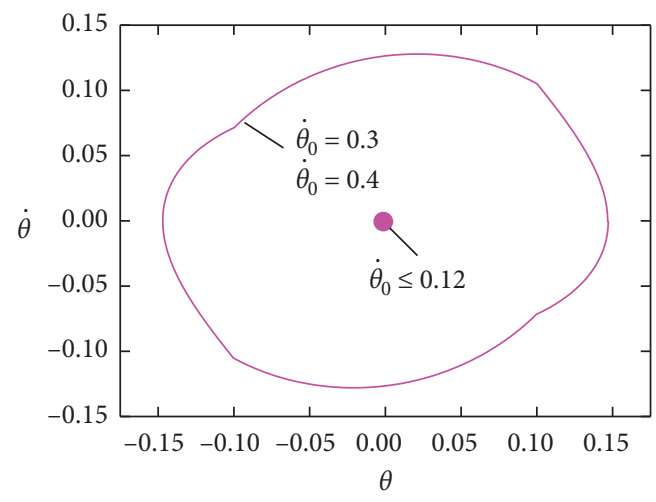

(c)

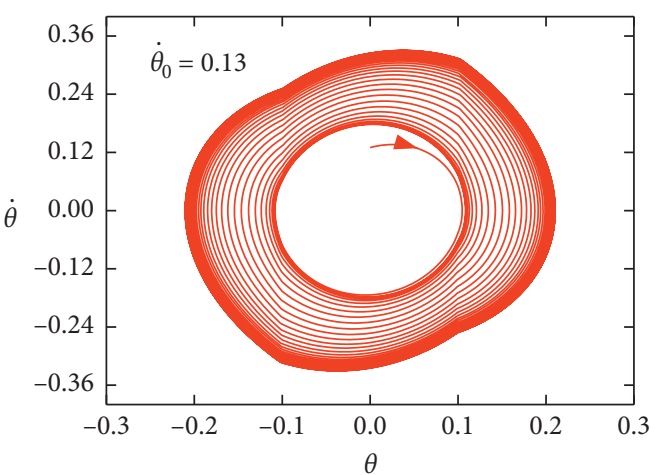

(b)

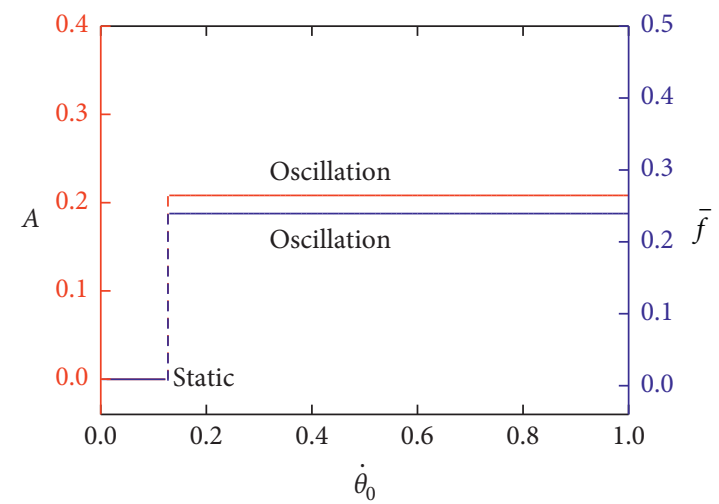

(d)

Figure 5: The effect of $\dot{\theta}_{0}$ on the swing of the simple pendulum, for $\theta_{0}=0, \varphi=0.1, \bar{\beta}=0.12, \varepsilon_{0}=0.3$, and $\bar{g}=2$. (a) Phase diagram of the static mode for $\dot{\theta}_{0}=0.12$. (b) Phase diagram of the oscillation mode for $\dot{\theta}_{0}=0.13$. (c) Limit cycles. (d) Amplitude and frequency.

stage of vibration, i.e., the pendulum is always in the illumination region. Therefore, the pendulum length $\bar{r}$ decreases continuously and approaches the value of $1-\varepsilon_{0.0}$. Figure $5(\mathrm{~b})$ plots the phase diagram of the swing angle for $\dot{\theta}_{0}=0.13$. The result shows that the amplitude of the single pendulum first increases and then asymptotically approaches a fixed value, which is oscillation mode. As explained previously, the single pendulum absorbs light autonomously during alternately swinging between the illumination and dark zones, which results in self-excited oscillation of the simple pendulum. Figure 5(c) plots the limit cycles, in which there exists a critical $\dot{\theta}_{0}=0.12$ for transition between static mode and oscillation mode.

Figure 5(d) plots the frequency and amplitude of selfexcited oscillation for different $\dot{\theta}_{0}$, respectively. For $\dot{\theta}_{0}>0.12$, the frequency and amplitude are both zero because the pendulum is in static mode. For $\dot{\theta}_{0}<0.12$, the frequency and amplitude of light-powered self-excited oscillation are the same and independent on $\dot{\theta}_{0}$. These results mean that the initial state affects the onset of the oscillation mode, while does not affect the frequency and amplitude of the oscillation. This is consistent with the general characteristic of self-excited motions [38].

4.2. Effect of Illumination Zone. Figure 6 shows the influence of the angle $\varphi$ of the illumination zone on the swing of the simple pendulum, for $\theta_{0}=0, \dot{\theta}_{0}=0.2, \varepsilon_{0}=0.3, \bar{\beta}=0.12$, and $\bar{g}=2$. Figure $6(\mathrm{a})$ plots the phase diagram of the swing angle of the pendulum, for $\varphi=0.154$. Initially, the maximum swing angle of the simple pendulum is larger than $\varphi$, and then, the simple pendulum swings with increasing amplitude alternately in the illuminated zone and the dark zone. The result shows that the oscillation mode of the pendulum is triggered. Figure 6(b) plots the phase diagram of the swing angle of the pendulum, for $\varphi=0.155$. The amplitude of the simple pendulum decreases continuously and the pendulum eventually comes to cease. This is because the pendulum with $\varphi$ larger than the maximum swing angle is always in the illumination zone and gradually develops into static mode. Figure 6(c) plots the limit cycles, in which there also exists a critical $\varphi$ for the phase transition between static mode and oscillation mode, which is numerically determined to be 0.154 .

Figure 6(d) plots the frequency and amplitude of selfexcited oscillation for different $\varphi$ values, respectively. For $\varphi>0.154$, the frequency and amplitude are both zero because the pendulum is in static mode. For $\varphi<0.154$, the frequency of light-powered self-excited oscillation is the same and independent on $\varphi$, while the amplitude is proportional to $\varphi$. These results mean that the illuminated zone affects the amplitude of the oscillation mode, while it does not affect the frequency of the oscillation. The result can be understood by the energy competition between energy input and damping dissipation. The increasing illuminated area 


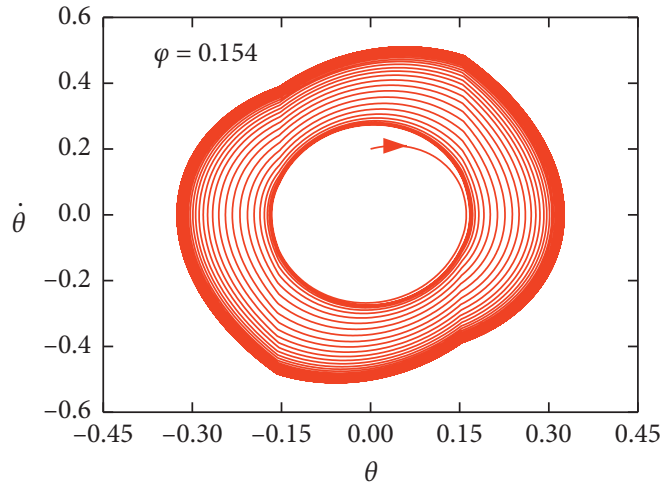

(a)

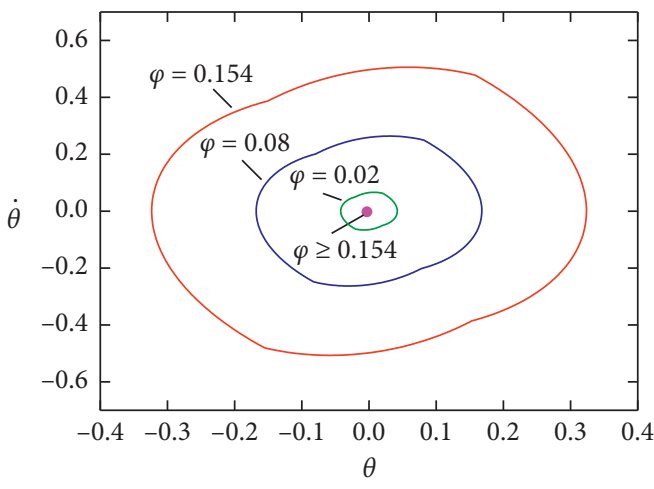

(c)

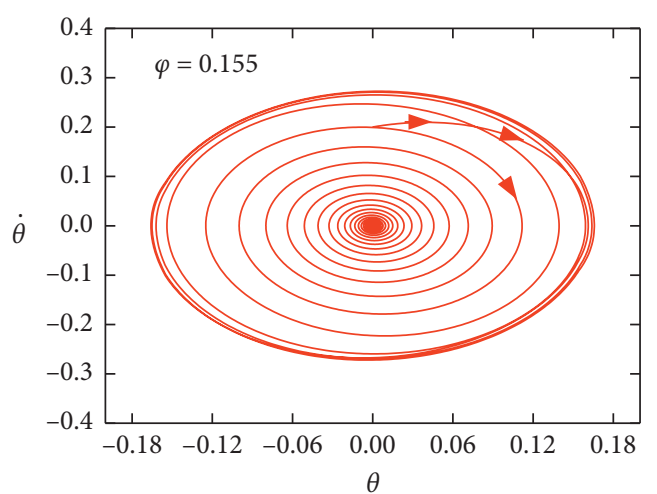

(b)

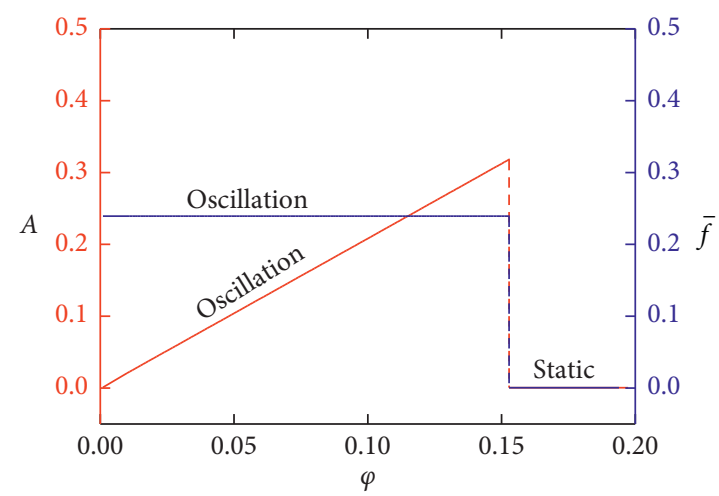

(d)

Figure 6: The effect of $\varphi$ on the swing of the pendulum, for $\theta_{0}=0, \dot{\theta}_{0}=0.2, \bar{\beta}=0.12, \varepsilon_{0}=0.3$, and $\bar{g}=2$. (a) Phase diagram of the oscillation mode for $\varphi=0.154$. (b) Phase diagram of the static mode for $\varphi=0.155$. (c) Limit cycles. (d) Amplitude and frequency.

amplifies the energy input and in turn enlarges the amplitude of the oscillation.

4.3. Effect of Damping Coefficient $\bar{\beta}$. Figure 7 shows the influence of $\bar{\beta}$ on the swing of the simple pendulum, for $\theta_{0}=0, \dot{\theta}_{0}=0.2, \varphi=0.1, \varepsilon_{0}=0.3$, and $\bar{g}=2$. Figure $7(\mathrm{a})$ plots the phase diagram of the swing angle of the pendulum, for $\bar{\beta}=0.15$. The result shows that the self-excited oscillation of the pendulum is triggered. For $\bar{\beta}=0.17$, the pendulum develops into static mode, as shown in Figure 7(b). These results can be understood by the energy competition between energy input and damping dissipation. With the increase of $\bar{\beta}$, the heat exchange between pendulum and environment becomes slower, and the net energy input during the swing decreases. Once the net energy input is not enough for compensating the damping dissipation, the pendulum will come to cease. Figure $7(\mathrm{c})$ plots the limit cycles, in which there also exists a critical $\bar{\beta}$ for the transition between static mode and oscillation mode, which is numerically determined to be 0.17 .

For small $\bar{\beta}$, the amplitude calculated may be larger than $\pi / 2$, i.e., the simple pendulum may rotate or the tension of the cycloid is negative, which is beyond the scope of this paper. Here, $\bar{\beta}$ is chosen as $\bar{\beta} \leq 0.02$. Figure $7(\mathrm{~d})$ plots the frequency and amplitude of self-excited oscillation for different $\bar{\beta}$, respectively. For $\bar{\beta}>0.17$, the frequency and amplitude are both zero because the pendulum is in static mode. For $\bar{\beta}<0.17$, the frequency of light-powered selfexcited oscillation increases slightly, while the amplitude decreases obviously and gradually approaches the fixed value, with the increase of $\bar{\beta}$. The result can also be understood by the energy competition between energy input and damping dissipation. The increasing $\bar{\beta}$ decreases the energy input and in turn attenuates the amplitude of the oscillation. The result implies that, in order to improve the absorption of light energy by the pendulum, $\bar{\beta}$ can be appropriately reduced for large energy harvesting in engineering.

4.4. Effect of Contraction Coefficient $\varepsilon_{0}$. Figure 8 shows the influence of $\varepsilon_{0}$ on the swing of the simple pendulum, for $\theta_{0}=0, \dot{\theta}_{0}=0.2, \varphi=0.1, \bar{\beta}=0.12$, and $\bar{g}=2$. Figure $8(\mathrm{a})$ plots the phase diagram of the swing angle of the pendulum, for $\varepsilon_{0}=0.22$. The pendulum is in static mode because the small strain of the cycloid cannot provide enough net energy input to compensate the energy dissipation by air damping. Figure 8 (b) plots the phase diagram of the swing angle of the pendulum, for $\varepsilon_{0}=0.25$. The result shows that the oscillation mode of the pendulum is triggered. In this case, the lightdriven strain can provide enough net energy input for the oscillation. Figure 8 (c) plots the limit cycles, in which there also exists a critical $\varepsilon_{0}$ for the phase transition between static mode and oscillation mode, which is numerically determined to be 0.25 . 


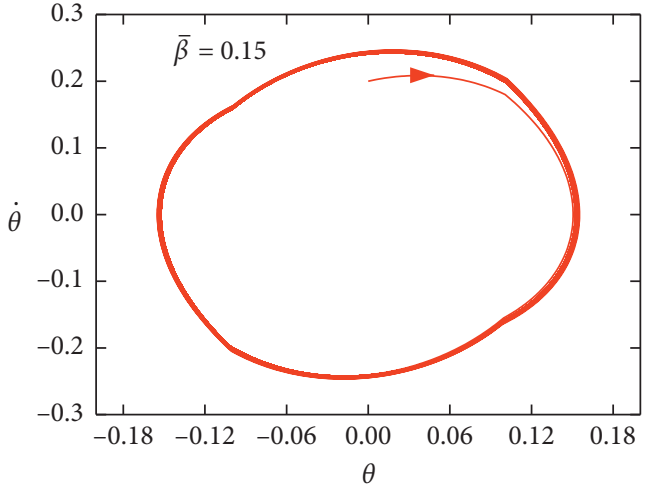

(a)

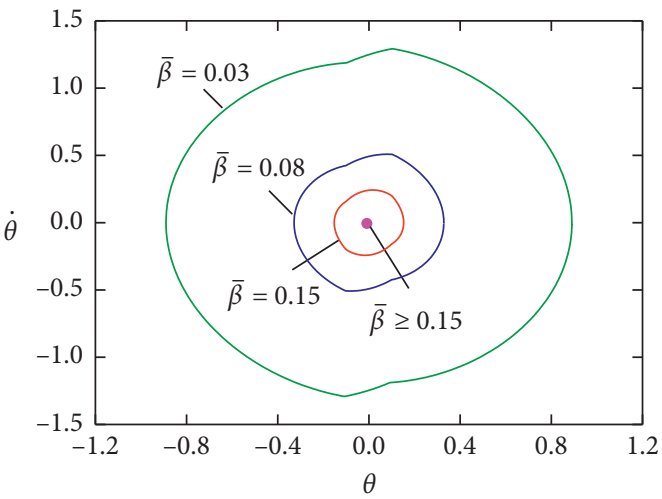

(c)

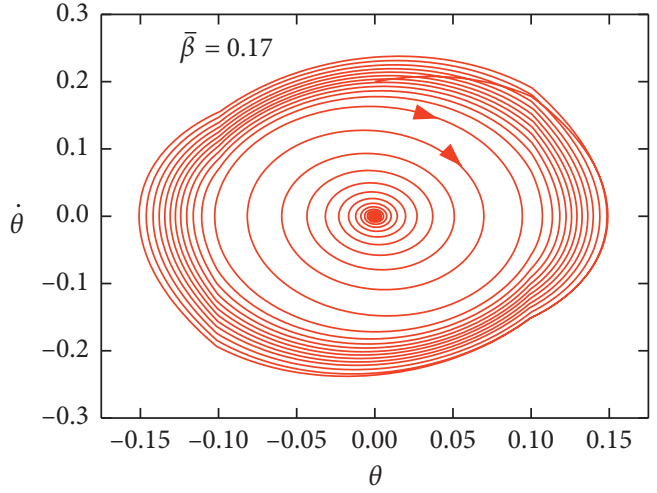

(b)

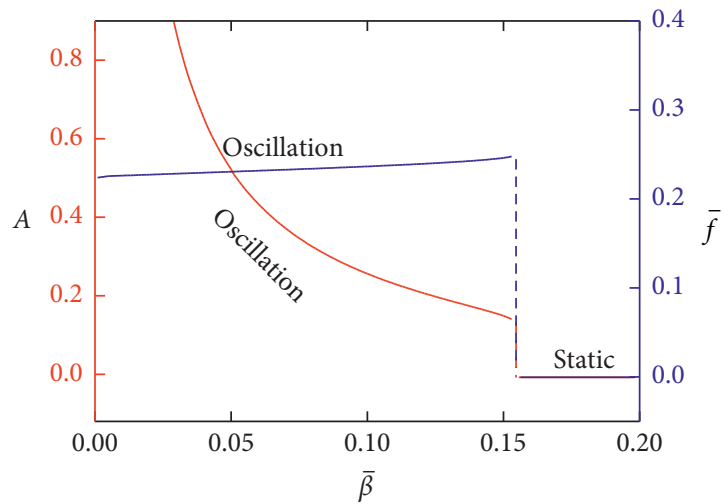

(d)

Figure 7: The effect of $\bar{\beta}$ on the swing of the pendulum for $\theta_{0}=0, \dot{\theta}_{0}=0.2, \varphi=0.1, \varepsilon_{0}=0.3$, and $\bar{g}=2$. (a) Phase diagram of the oscillation mode for $\bar{\beta}=0.15$. (b) Phase diagram of the static mode for $\bar{\beta}=0.17$. (c) Limit cycles. (d) Amplitude and frequency.

For larger $\varepsilon_{0}$, the pendulum can also oscillate continuously. However, according to the physical meaning of contraction strain $\varepsilon_{0}$, the maximum value should be smaller than 1 . Here, $\varepsilon_{0}$ is chosen as $0.25 \leq \varepsilon_{0} \leq 0$.9. Figure $8(\mathrm{~d})$ plots the frequency and amplitude of self-excited oscillation for different $\varepsilon_{0}$, respectively. For $\varepsilon_{0}<0.25$, the frequency and amplitude are both zero because the pendulum is in static mode. For $\varepsilon_{0}>0.25$, the increase of $\varepsilon_{0}$ has no obvious effect on the self-excited oscillation frequency, while the amplitude increases dramatically with the increase of $\varepsilon_{0}$. Therefore, in order to improve the engineering application of photothermal-responsive pendulum, light-driven contractile or expansive strain of the material should be amplified for the efficient conversion of light energy to mechanical energy.

4.5. Effect of Gravitational Acceleration $\bar{g}$. Figure 9 shows the influence of $\bar{g}$ on the swing of the simple pendulum, for $\theta_{0}=0, \dot{\theta}_{0}=0.2, \varphi=0.1, \bar{\beta}=0.12$, and $\varepsilon_{0}=0.3$. Figure 9 (a) plots the phase diagram of the swing angle of the pendulum for $\bar{g}=0.4$, which is static mode. Figures 9 (b) and 9(c) plot the phase diagrams of the swing angle of the pendulum, for $\bar{g}=0.5$ and $\bar{g}=4.7$, respectively. The result shows that the oscillation mode of the pendulum is triggered. Careful calculations show that, for $0.4 \leq \bar{g} \leq 4.7$, the simple pendulum is in oscillation mode. However, for $\bar{g}=4.8$, the pendulum swings in static mode again, as shown in Figure 9(d). Figure 9(e) also plots the limit cycles of the light-powered swing of the pendulum. The results can be understood by the energy compensation between net energy input and damping dissipation. For small $\bar{g}$, i.e., the heat exchange is too fast, the cycloid rapidly deforms just only when passing through the interface between the illumination and dark zones. For large $\bar{g}$, i.e., the heat exchange is too slow, the cycloid almost does not deform during the swing. Therefore, the net work done of single pendulum by the light is too small and cannot compensate the energy dissipated by air damping to maintain the oscillation.

Figure 9(f) plots the frequency and amplitude of selfexcited oscillation of the simple pendulum for $0.4 \leq \bar{g} \leq 4.7$, respectively. It can be seen that the oscillation frequency and amplitude of the pendulum can be significantly increased by increasing $\bar{g}$. According to the physical meaning of $\bar{g}$, reducing the heat transfer rate within a certain range can amplify the oscillation frequency and amplitude and improve the efficiency of light energy to mechanical energy in engineering application. 


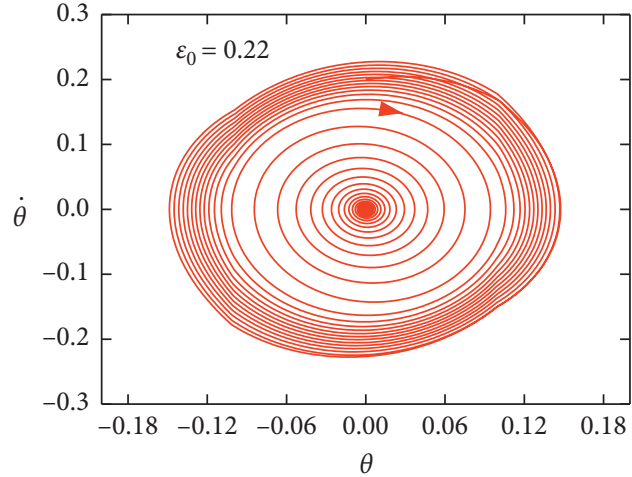

(a)

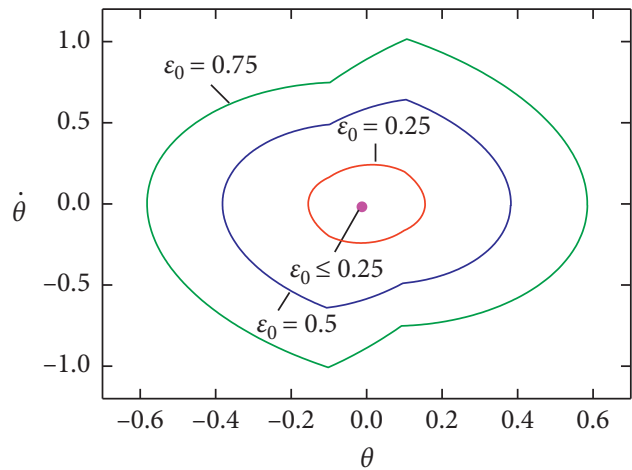

(c)

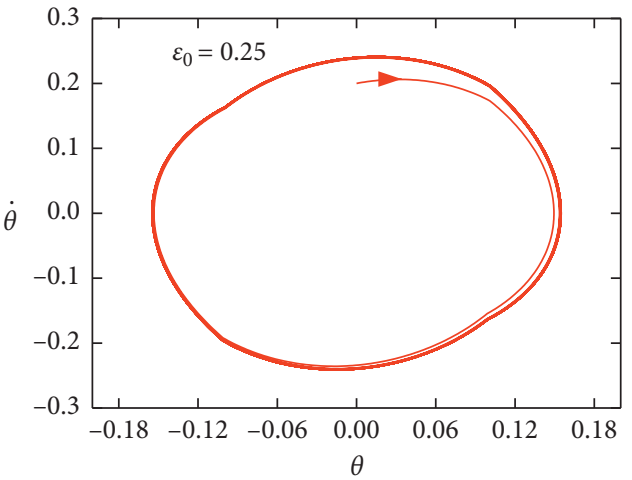

(b)

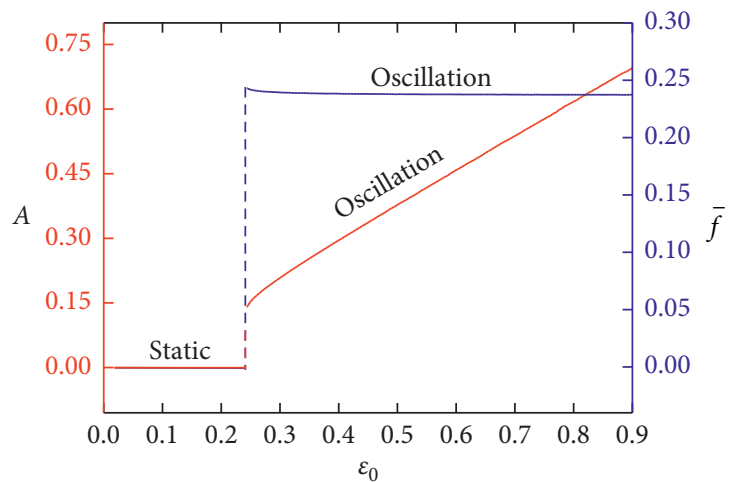

(d)

Figure 8: The effect of $\varepsilon_{0}$ on the swing of the pendulum for $\theta_{0}=0, \dot{\theta}_{0}=0.2, \varphi=0.1, \bar{\beta}=0.12$, and $\bar{g}=2$. (a) Phase diagram of the static mode at $\varepsilon_{0}=0.22$. (b) Phase diagram of the oscillation mode at $\varepsilon_{0}=0.25$. (c) Limit cycles. (d) Amplitude and frequency.

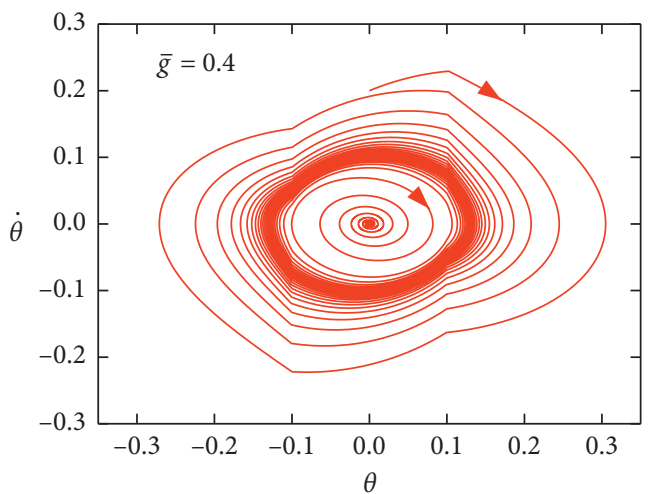

(a)

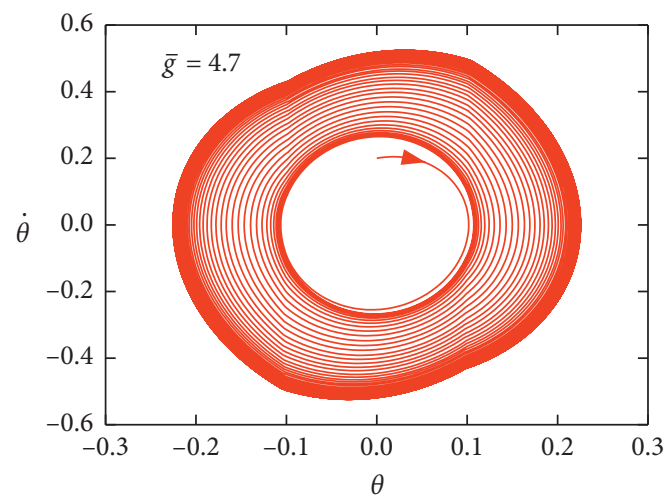

(c)

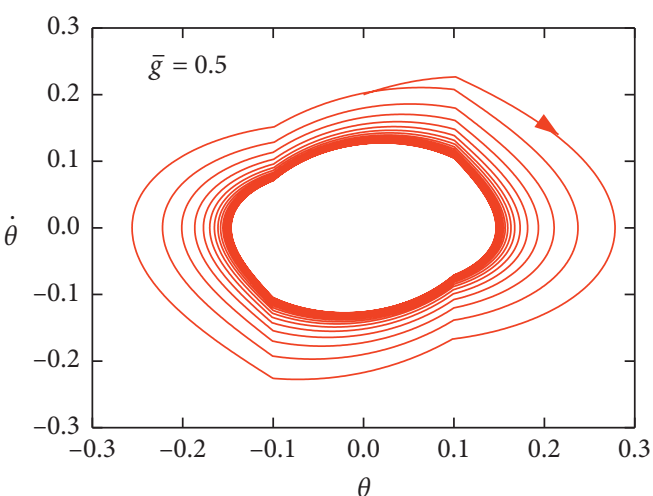

(b)

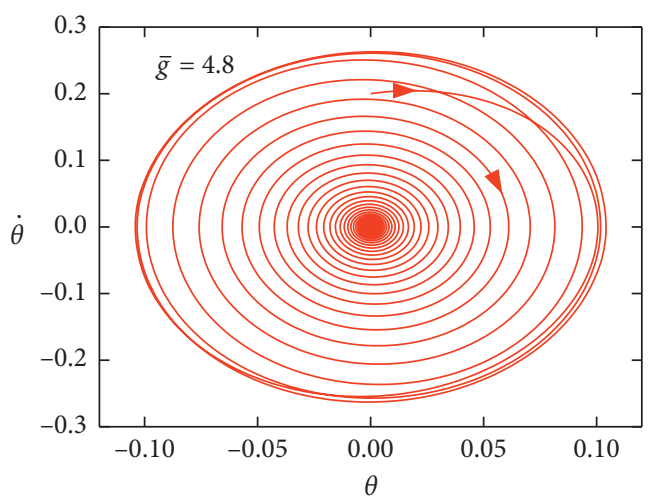

(d)

Figure 9: Continued. 


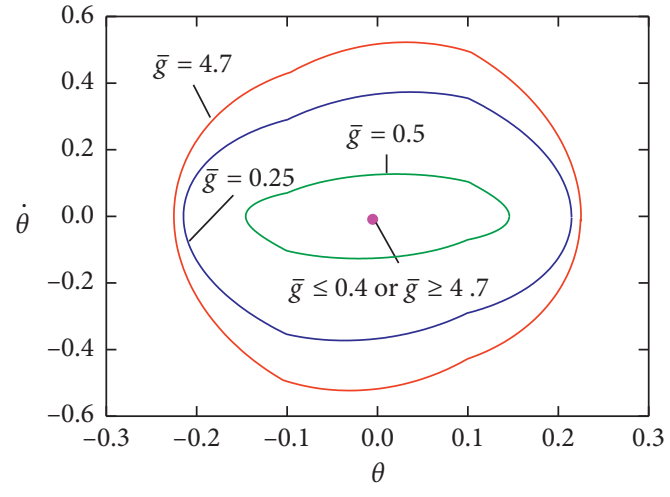

(e)

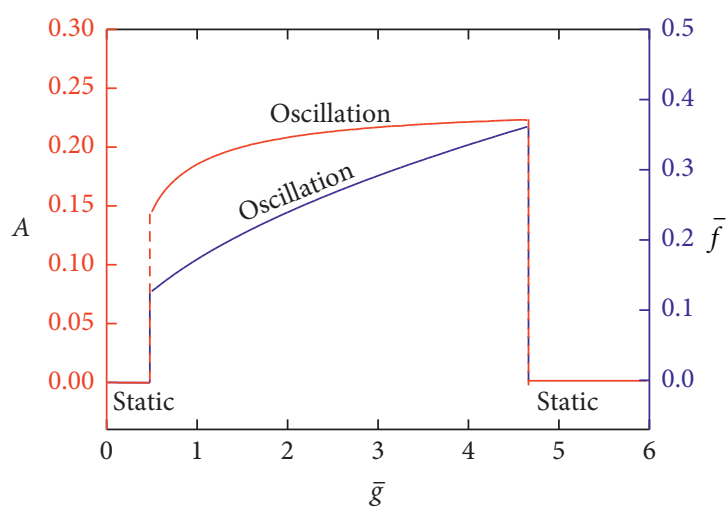

(f)

Figure 9: The effect of $\bar{g}$ on the swing of the pendulum for $\theta_{0}=0, \dot{\theta}_{0}=0.2, \varphi=0.1, \bar{\beta}=0.12$, and $\varepsilon_{0}=0.3$. (a) Phase diagram of the static mode at $\bar{g}=0.4$. (b) Phase diagram of the oscillation mode at $\bar{g}=0.5$. (c) Phase diagram of the oscillation mode at $\bar{g}=4.7$. (d) Phase diagram of the static mode at $\bar{g}=4.8$. (e) Limit cycles. (f) Amplitude and frequency.

\section{Conclusions}

Photothermal-responsive materials can convert light energy into mechanical energy for driving active machines. In this work, a simple pendulum capable of self-excited oscillation is innovatively constructed by using ordinary PSM or PEM as the cycloid, and the dynamic model is established for investigating the light-powered swing of the photothermal-responsive pendulum. By theoretical formulation and numerical calculation, the self-excited oscillation behaviors of the simple pendulum are extensively studied. When the PSM pendulum is irradiated in the central zone of the swing, or conversely the PEM pendulum is illuminated on the two sides of the swing, the simple pendulum could oscillate autonomously. There exist two motion modes of the pendulum, which are static mode and oscillation mode. The numerical calculation provides time histories of the cycloid length and cycloid tension in detail and in turn elucidates the mechanism of self-excited oscillation that results from the coupling of heat exchange, photothermal deformation, and swing. When the net input energy compensates the damping dissipation, the pendulum keeps swinging. This paper further investigates the influence of physical parameters on the self-excited oscillation. The results show that the amplitude of the selfexcited oscillation can be effectively increased by increasing the illumination zone, reducing the air damping dissipation, increasing the limit contraction strain of the material, or decreasing the heat transfer rate of the photothermal material, while the frequency of the self-excited oscillation is almost only affected by the heat transfer coefficient of the photothermal-responsive material. Unfortunately, as far as we know, there are no experimental studies in the literature to verify our findings. Next, we do plan to carry out corresponding experiments to further confirm our results. We hope the light-powered self-excited oscillation of photothermal-responsive pendulums can not only deepen the understanding of self-excited oscillations but also have potential applications in harvesting energy, signal sensing, soft robots, and so on.

\section{Data Availability}

No data were used to support the findings of this study.

\section{Conflicts of Interest}

The authors declare no conflicts of interest.

\section{Acknowledgments}

This study was supported by the Outstanding Talents Cultivation Project of Universities in Anhui (Grant no. gxyqZD2019056) and the Key Project of Natural Science Research of Universities in Anhui (Grant nos. KJ2020A0449 and KJ2020A0452).

\section{References}

[1] A. Suzuki and T. Tanaka, "Phase transition in polymer gels induced by visible light," Nature, vol. 346, no. 6282, pp. 345-347, 1990.

[2] Z. Hu, Y. Li, X. Zhang, and C. L. Littler, " $\mathrm{CO}_{2}$ laser-controlled transmission of visible light in $\mathrm{N}$-isopropylacrylamide gel," Polymer Gels and Networks, vol. 3, no. 3, pp. 267-279, 1995.

[3] S. Kubo, Z.-Z. Gu, K. Takahashi, A. Fujishima, H. Segawa, and O. Sato, "Control of the optical properties of liquid crystalinfiltrated inverse opal structures using photo irradiation and/ or an electric field," Chemistry of Materials, vol. 17, no. 9, pp. 2298-2309, 2005.

[4] M. H. Li and P. Keller, "Artificial muscles based on liquid crystal elastomers," Philosophical Transactions of the Royal Society A-Mathematical Physical and Engineering Sciences, vol. 364, no. 1847, pp. 2763-2777, 2006.

[5] N. J. Dawson, M. G. Kuzyk, J. Neal, P. Luchette, and P. PalffyMuhoray, "Cascading of liquid crystal elastomer photomechanical optical devices," Optics Communications, vol. 284, no. 4, pp. 991-993, 2011.

[6] M. Camacho-Lopez, H. Finkelmann, P. Palffy-Muhoray, and M. Shelley, "Fast liquid-crystal elastomer swims into the dark," Nature Materials, vol. 3, no. 5, pp. 307-310, 2004.

[7] C. Huang, J. A. Lv, X. Tian, Y. Wang, Y. Yu, and J. Liu, "Miniaturized swimming soft robot with complex movement 
actuated and controlled by remote light signals," Scientific Reports, vol. 5, Article ID 17414, 2015.

[8] L. Yu and $\mathrm{H}$. Yu, "Light-powered tumbler movement of graphene oxide/polymer nanocomposites," ACS Applied Materials \& Interfaces, vol. 7, no. 6, pp. 3834-3839, 2015.

[9] J. A. Rogers, T. Someya, and Y. Huang, "Materials and mechanics for stretchable electronics," Science, vol. 327, no. 5973, pp. 1603-1607, 2010.

[10] F. Cheng, R. Yin, Y. Zhang, C.-C. Yen, and Y. Yu, "Fully plastic microrobots which manipulate objects using only visible light," Soft Matter, vol. 6, no. 15, pp. 3447-3449, 2010.

[11] T. Ikeda, J.-i. Mamiya, and Y. Yu, "Photomechanics of liquidcrystalline elastomers and other polymers," Angewandte Chemie International Edition, vol. 46, no. 4, pp. 506-528, 2007.

[12] S.-H. Hu, R.-H. Fang, Y.-W. Chen, B.-J. Liao, I.-W. Chen, and S.-Y. Chen, "Photoresponsive protein-graphene-protein hybrid capsules with dual targeted heat-triggered drug delivery approach for enhanced tumor therapy," Advanced Functional Materials, vol. 24, no. 26, pp. 4144-4155, 2014.

[13] Y. Zeng and J. Q. Lu, "Optothermally responsive nanocomposite generating mechanical forces for cells enabled by few-walled carbon nanotubes," ACS Nano, vol. 8, no. 11, pp. 11695-11706, 2014.

[14] Y. Yang, W. Zhan, R. Peng et al., "Graphene-Enabled superior and tunable photomechanical actuation in liquid crystalline elastomer nanocomposites," Advanced Materials, vol. 27, no. 41, pp. 6376-6381, 2015.

[15] C. Li, Y. Liu, C.-w. Lo, and H. Jiang, "Reversible white-light actuation of carbon nanotube incorporated liquid crystalline elastomer nanocomposites," Soft Matter, vol. 7, no. 16, pp. 7511-7516, 2011.

[16] L. Q. Yang, K. Setyowati, A. Li, Sh. Q. Gong, and J. Chen, "Reversible infrared actuation of carbon nanotube-liquid crystalline elastomer nanocomposites," Advanced Materials, vol. 20, no. 12, pp. 2271-2275, 2010.

[17] Y. Ji, Y. Y. Huang, R. Rungsawang, and E. M. Terentjev, "Dispersion and alignment of carbon nanotubes in liquid crystalline polymers and elastomers," Advanced Materials, vol. 22, no. 31, pp. 3436-3440, 2010.

[18] J. E. Marshall, Y. Ji, N. Torras, K. Zinoviev, and E. M. Terentjev, "Carbon-nanotube sensitized nematic elastomer composites for ir-visible photo-actuation," Soft Matter, vol. 8, no. 5, pp. 1570-1574, 2012.

[19] S. C. Chang, "Stability, chaos detection, and quenching chaos in the swing equation system," Mathematical Problems in Engineering, vol. 2020, Article ID 6677084, 12 pages, 2020.

[20] H. Zeng, M. Lahikainen, L. Liu et al., "Light-fuelled freestyle self-oscillators," Nature Communications, vol. 10, no. 1, pp. 1-9, 2019.

[21] K. Li, X. Su, and S. Q. Cai, "Self-sustained rolling of a thermally responsive rod on a hot surface," Extreme Mechanics Letters, vol. 42, Article ID 101116, 2021.

[22] A. Chakrabarti, G. P. Choi, and L. Mahadevan, "Self-excited motions of volatile drops on swellable sheets," Physical Review Letters, vol. 124, no. 25, Article ID 258002, 2020.

[23] B. Ramakrishnan, R. Ramamoorthy, C. Li, A. Akgul, and K. Rajagopal, "Spiral waves in a lattice array of Josephson junction chaotic oscillators with flux effects," Mathematical Problems in Engineering, vol. 2021, Article ID 8848914, 9 pages, 2021

[24] Y. Xu, W. Hu, and S. P. Zhang, "Dynamic simulation of a woodpecker robot based on self-excited vibration," Advanced Materials Research, vol. 753-755, pp. 2020-2024, 2013.
[25] K. Ono, T. Furuichi, and R. Takahashi, "Self-excited walking of a biped mechanism," International Journal of Robotics Research, vol. 23, no. 1, pp. 953-966, 2001.

[26] I. Morazzani, D. Lahr, D. Hong, P. Ren, and V. Tech, "Novel tripedal mobile robot and considerations for gait planning strategies based on kinematics," Lecture Notes in Control and Information Sciences, vol. 370, pp. 35-48, 2008.

[27] C. Li and X. He, "A bio-mimetic pipe crawling microrobot driven based on self-excited vibration," in Proceedings of the IEEE International Conference on Robotics And Biomimetics, pp. 984-988, Sanya, China, 2007.

[28] A. Yamano and H. Ijima, "Basic study of the adaptive control for the swimming robot using self-excited oscillation," Transactions of the JSME (In Japanese), vol. 84, no. 864, pp. 18-00054, 2018.

[29] N. A. D. Stokes, R. M. A. Fatah, and S. Venkatesh, "Selfexcitation in fibre-optic microresonator sensors," Sensors and Actuators A Physical, vol. 21, no. 1-3, pp. 369-372, 1990.

[30] K. Hane and K. Suzuki, "Self-excited vibration of a selfsupporting thin film caused by laser irradiation," Sensors and Actuators A: Physical, vol. 51, no. 2-3, pp. 179-182, 1995

[31] K. Aubin, M. Zalalutdinov, T. Alan et al., "Limit cycle oscillations in cw laser-driven nems," Journal of Microelectromechanical Systems, vol. 13, no. 6, pp. 1018-1026, 2004.

[32] H. Hoelscher, P. U. Zerweck, L. M. Eng, and R. Hoffmann, "The effective quality factor at low temperatures in dynamic force microscopes with Fabry-Pérot interferometer detection," Applied Physics Letters, vol. 94, no. 22, Article ID 223514, 2009.

[33] C. Metzger, M. C. Neuenhahn, A. Ortlieb, I. Favero, K. Karrai, and F. Marquardt, "Self-induced oscillations in an optomechanical system driven by bolometric backaction," Physical Review Letters, vol. 101, no. 13, Article ID 133903, 2008.

[34] E. Hollander and O. Gottlieb, "Self-excited chaotic dynamics of a nonlinear thermo-visco-elastic system that is subject to laser irradiation," Applied Physics Letters, vol. 101, no. 13, Article ID 133507, 2012.

[35] A. H. Gelebart, G. Vantomme, E. W. Meijer, and D. J. Broer, "Mastering the photothermal effect in liquid crystal networks: a general approach for self-sustained mechanical oscillators," Advanced Materials, vol. 29, no. 18, Article ID 1606712, 2017.

[36] S. Houri, S. J. Cartamil-Bueno, M. Poot, P. G. Steeneken, H. S. J. van der Zant, and W. J. Venstra, "Direct and parametric synchronization of a graphene self-oscillator," Applied Physics Letters, vol. 110, no. 7, Article ID 073103, 2017.

[37] E. Buks and I. Martin, "Self-excited oscillation and synchronization of an on-fiber optomechanical cavity," Physical Review E, vol. 100, no. 3, Article ID 032202, 2019.

[38] W. Ding, Self-excited Vibration, Springer, Berlin, Germany, 2010. 